\title{
Determination of land surface temperature and soil moisture from Tropical Rainfall Measuring Mission/Microwave Imager remote sensing data
}

\author{
Jun Wen ${ }^{1}$ and Zhongbo Su \\ Wageningen University and Research Centre, Alterra Green World Research, Wageningen, Netherlands \\ Yaoming Ma \\ Cold and Arid Regions Environmental and Engineering Research Institute, Chinese Academy of Sciences, Lanzhou, China \\ Received 6 February 2002; revised 7 September 2002; accepted 11 September 2002; published 16 January 2003.
}

[1] An analytical algorithm for the determination of land surface temperature and soil moisture from the Tropical Rainfall Measuring Mission/Microwave Imager (TRMM/TMI) remote sensing data has been developed in this study. The error analyses indicate that the uncertainties of the enrolled parameters will not cause serious errors in the proposed algorithm. By applying the proposed algorithm to TRMM/TMI remote sensing data collected during the Global Energy and Water Experiment (GEWEX) Asian Monsoon Experiment (GAME)/Tibet Intensive Observation Period field campaign in 1998 (IOP'98), the temporal and regional distributions of land surface temperature and volumetric soil moisture are evaluated over the central Tibetan plateau area. To validate the proposed method, the ground-measured surface temperature and volumetric soil moisture are compared to TRMM/TMI-derived land surface temperature and soil Fresnel reflectivity respectively. The results show that the estimated surface temperature is in good agreement with ground measurements; their difference and correlation coefficient are $0.52 \pm 2.41 \mathrm{~K}$ and 0.80 , respectively. A quasi-linear relationship exists between estimated Fresnel reflectivity and ground-measured volumetric soil moisture with a correlation coefficient 0.82 . The land surface thermal status can also be clearly identified from the regional distribution of the estimated land surface temperature; the mountainous area and water bodies have a very lower surface temperature, while the river basin shows a higher surface temperature compared to the mountainous area. The southeastern part of the selected area has lower soil moisture, while the river basin exhibits high soil moisture. It is therefore concluded that the proposed algorithm is successful for the retrieval of land surface temperature and soil moisture using TRMM/TMI data over the study

area. INDEX TERMS: 1640 Global Change: Remote sensing; 1866 Hydrology: Soil moisture; 3322 Meteorology and Atmospheric Dynamics: Land/atmosphere interactions; KEYWORDS: TRMM/TMI, brightness temperature, Tibetan plateau, land surface temperature and soil moisture

Citation: Wen, J., Z. Su, and Y. Ma, Determination of land surface temperature and soil moisture from Tropical Rainfall Measuring Mission/Microwave Imager remote sensing data, J. Geophys. Res., 108(D2), 4038, doi:10.1029/2002JD002176, 2003.

\section{Introduction}

[2] Asia is considerably affected by severe drought and flood disasters caused by the anomalous precipitation mainly associated with the annual climate of Asia monsoon. Asia monsoon influences the atmospheric dynamic and thermal processes over the Tibetan plateau, especially its heating and cooling effects [Yanai and $L i, 1994]$. On the other hand, land surface soil moisture integrates the local

\footnotetext{
${ }^{1}$ Also at Cold and Arid Regions Environmental and Engineering Research Institute, Chinese Academy of Sciences, Lanzhou, China.

Copyright 2003 by the American Geophysical Union. 0148-0227/03/2002JD002176
}

precipitation and surface evaporation over the Tibetan plateau, which in turn influences the land-atmospheric energy exchange, the weather and climatic variation in eastern Asia. Therefore knowledge about soil moisture distribution over the Tibetan plateau and its retrieval algorithm will be necessary to improve the understanding of Asia monsoon.

[3] Satellite or airborne remote sensing sensor or instrument can continuously observe the earth surface from space; these observation data have been widely used in many studies and application fields. Many attempts on the determination of land surface parameters have been successfully achieved by using satellite remote sensing data [Tucker and Sellers, 1986; Ormsby and Choudhury, 1987; Becker and Choudhury, 1988; Asrar et al., 1989; Su, 
2000]. To correct atmospheric effects existing between satellite sensor and land surface or to remove cloud contamination are still the difficult tasks in optical and infrared remote sensing in the determination of land surface parameters. Microwave radiation can penetrate atmospheric layer with little attenuation at lower frequency or longer wavelength band, there is therefore an increasing trend in determination of the land surface parameters or monitoring land surface properties from microwave remote sensing data [Wigneron et al., 1993]. Similar to infrared remote sensing process, passive microwave radiometer receives radiance emitted from the earth surface, which can be converted to surface brightness temperature. Brightness temperature is an integration of land surface configurations, such as land surface soil moisture, surface roughness, surface physical temperature and the contribution from vegetation layer over vegetated surfaces [Njoku and Kong, 1977]. With dual-polarized and multiple-channel brightness temperature observation data, it is possible to retrieve soil moisture and surface temperature simultaneously. It is also possible to extract vegetation information or to estimate vegetation parameters based on the difference between the polarized land surface brightness temperatures [Calvet et al., 1996].

[4] The Tropical Rainfall Measuring Mission (TRMM) carries onboard several remote sensors such as Precipitation Radar (PR), Microwave Imager (TMI), Visible and Infrared radiometer Scanner (VIRS), Clouds and Earth's Radiant Energy System (CERES) and Lightning Image System (LIS). TMI is a microwave radiometer with multi-channels in dual-polarization including $10.7 \mathrm{GHz}, 19.4 \mathrm{GHz}, 37.0 \mathrm{GHz}$ and $85.5 \mathrm{GHz}$, and $21.3 \mathrm{GHz}$ channel only in vertical polarization. The lower frequency data have been successfully used to retrieve land surface vegetation [Wen and $\mathrm{Su}, 2001$ ] and soil moisture [Fujii and Koike, 2001].

[5] The major efforts in TRMM/TMI research have being focused on the quantitative retrieval of the tropic precipitation. In this contribution, an algorithm for the determination of land surface temperature and soil moisture with 10.7 GHz, $19.4 \mathrm{GHz}$ dual-polarization and $21.3 \mathrm{GHz}$ monovertical polarization TRMM/TMI observation data has been successfully developed, which is a new application field for TRMM/TMI data. The algorithm is physically based on the radiative transfer process and an advanced solution for the no-linear equations system. It will be described in detail in the second section. The error analysis, validation and comparison between ground measurements and retrieved results will be presented subsequently. The relationship between TRMM/TMI retrieved Fresnel reflectivity and ground-measured volumetric soil moisture has been established. Then this relationship has been extended to an application in the regional scale, the regional distributions of land surface temperature and soil moisture and discussion are presented. The conclusions of this study will be drawn in the last section.

\section{Methodology}

[6] In the microwave propagation media, atmosphere and vegetation layer, no distinct border exists between the top of vegetation and the bottom of atmosphere layer. At $p$ polarization with an incidence angle $\theta$, surface brightness temper- ature over the vegetated land surface can be expressed as follows [Ulaby et al., 1982]

$$
T_{B p}(\tau, \theta)=\left(1+L \Gamma_{p}\right)(1-L)(1-\omega) T_{V}+\left(1-\Gamma_{p}\right) L T_{S},
$$

where the first term at the right hand side of this equation is contribution from vegetation layer, the second term is contribution from soil. $T_{B p}$ is the brightness temperature at $p$ polarization, which refers to $h$ or $v . T_{V}$ and $T_{S}$ are the vegetation and soil effective temperature respectively, $\Gamma_{\mathrm{p}}$ is soil reflectivity at $p$ polarization, $L$ is canopy attenuation given by $L=\exp (-m \tau), m$ is relative atmosphere mass along the radiative transfer path given by $m=\sec (\theta), \tau$ is the vegetation opacity or optical thickness, which depends on canopy extinction coefficient and canopy height $\left(\tau=\kappa_{\mathrm{e}} \mathrm{H}\right)$, $\omega$ is single scattering albedo of the vegetation layer, $e_{s p}=$ $\left(1-\Gamma_{p}\right)$ is soil emissivity at $p$ polarization. Above equation has been widely used in determination of soil wetness and vegetation parameters. The vegetation contributions consist of two parts: the upward radiance directly emitted from vegetation layer toward satellite sensor, $(1-\omega)(1-L) T_{V}$, and the downward radiance emitted from vegetation layer toward to ground and then reflected by the land surface and transmitted through vegetation layer to satellite sensor, $T_{V} \Gamma_{s} L(1-\omega)(1-L)$. The later part is usually very small in general case because of the smaller magnitudes of surface reflectivity and vegetation attenuation, if this term could be neglected, equation (1) can be rewritten as following equation

$$
T_{B p}(\tau, \theta)=(1-\omega)(1-L) T_{V}+\left(1-\Gamma_{p}\right) L T_{S}
$$

[7] As land surface brightness temperatures are polarization variant because of the radiative propagation property, soil and vegetation water content [Van de Griend and Owe, 1994], Normalized Difference Polarization Index (NDPI) can be configured as following, which is also an indicator of the surface vegetation information and soil moisture or wetness

$$
P I(\tau, \theta)=\frac{T_{B v}-T_{B h}}{0.5 \cdot\left(T_{B v}+T_{B h}\right)}
$$

[8] In most case, the single scattering albedo ranges from about 0.03 to 0.08 for the different vegetation types at 10 $\mathrm{GHz}$ [Chanzy and Wigneron, 2000], the soil emissivity at the corresponding frequency is about 0.85-0.95 [Matzler and Wiesmann, 2000]. For the sparsely vegetated land surface cases, vegetation layer and surface soil have equivalent temperatures especially in the morning or evening hours. By combining equations (2) and (3), we can derive Normalized Difference Polarization Index as following formula

$$
P I(\tau, \theta)=P I(0, \theta) \exp (-\tau \sec \theta)
$$

where $P I(0, \theta)$ is NPDI for bare soil. The optical thickness of canopy layer is a function of plant water content $(P W C$ in $\mathrm{kg} / \mathrm{m} 2)$, leaf area index ( $L A I$ in $\mathrm{m} 2 / \mathrm{m} 2)$ and wavelength, $P I(\tau, \theta)$ at both $\mathrm{X}$ - and $\mathrm{Ka}$-bands is related to the vegetation biomass of vegetation type through these two parameters: 
$P W C$ and $L A I$. Since a logarithmic relationship exists among these parameters [Paloscia et al., 2001], LAI can be derived as following equation

$$
L A I=\frac{v \cdot \mu \cdot \sqrt{\lambda}}{\kappa} \ln \left[\frac{P I(0, \theta)}{P I(\tau, \theta)}\right],
$$

where $v$ is an empirical correlation factor between plant water content and leaf area index, the typical value is 3.3 for corn, 1.0 is assumed for the natural grass in this study. $\mu=$ $\cos (\theta) . \kappa$ is a factor depending on the vegetation type, the value is $0.1 \mathrm{~m}^{1 / 2}$ for sunflower and $0.4 \mathrm{~m}^{1 / 2}$ for alfalfa, $\mathrm{m}$ is the length unit meter. For the natural vegetation in the Tibet plateau, $0.3 \mathrm{~m}^{1 / 2}$ is assigned as a guess value for $\kappa$ in this contribution.

[9] Wen et al. (J. Wen and Z. Su, An analytical algorithm on the determination of LAI from TRMM/TMI data, submitted to International Journal of Remote Sensing, 2001.) reported that regional distribution of TRMM/TMI estimated LAI is in agreement with the regional and temporal distribution of NOAA/AVHRR-derived Normalized Difference Vegetation Index. Therefore this scheme for land surface vegetation parameterization is reliable. As an extensive application, the regional leaf area index can be derived from $10.65 \mathrm{GHz}$ dual polarization data when TRMM/TMI overpasses the study area. The estimated LAI will be used to evaluate land surface temperature and soil moisture from the proposed algorithm.

[10] In order to derive land surface temperature and soil moisture, TRMM/TMI dual-polarized brightness temperature at $19.35 \mathrm{GHz}$ and vertically polarized brightness at $21.3 \mathrm{GHz}$ channel will be indispensable in this study. Brightness temperature is an integration of land surface temperature, soil moisture, surface roughness and vegetation contribution. Surface roughness is a time invariant variable characterized by standard deviation of surface height for natural surfaces, its regional distribution can be evaluated from ERS Wind scatterometer data [Wen and Su, 2001]. ERS Wind scatterometer-derived roughness variable is the root mean square (RMS) slope of land surface height; the roughness variable in Equation (7) is the standard deviation of land surface height. If the root mean square slope of land surface height is available, it can be easily converted to the standard deviation of land surface height with the known correlation length of $10 \mathrm{~cm}$ reported for the Tibetan plateau by Tadono et al. [1999]. With the estimated LAI, if the surface reflectivity is assumed equal at 19.35 $\mathrm{GHz}$ and $21.3 \mathrm{GHz}$ channels because these two frequencies are very close, with the three channels' observation data and equation (1), it will configure a no-linear equation system. Three unknown variables: surface temperature and soil reflectivity in vertical and horizontal polarization can be retrieved from this nonlinear equation system with an advanced numerical root finding solution [William et al., 1992]. As the effects of surface roughness has been integrated into the derived soil reflectivity in equation (2), an effective method for separating Fresnel reflectivity and surface roughness is also necessary. To this end, Choudhury et al. [1979] proposed a simple model based on the soil Fresnel reflectivity and the standard deviation of surface height to characterize soil reflectivity. Wang and Choudhury [1981] improved the formulation and the relationship between land surface reflectivity and roughness variable as following equation

$$
\Gamma_{p}=\left[(1-Q) \Gamma_{s p}+Q \Gamma_{s q}\right] \exp \left(-k^{2} \sigma^{2} \cos ^{2} \theta\right),
$$

where $\Gamma$ is the reflectivity of rough surface, $p$ ( $h$ or $v$ ) is polarization and the polarization orthogonal to $q, s$ means the smooth land surface, $\sigma$ is the standard deviation of surface height, $\theta$ is incidence angle of radiation beam, $Q$ can be simply determined as expression

$$
Q=0.35\left[1-\exp \left(-0.66 \sigma^{2} f\right)\right]
$$

where $f$ is frequency of the microwave radiation beam in GHz [Kerr and Njoku, 1990]. Equations (6) and (7) will be adopted to take into account the relationship between the land surface roughness and soil Fresnel reflectivity in this study.

[11] The surface soil horizontal Fresnel reflectivity exhibits an approximately linear dependence on volumetric soil moisture

$$
\Gamma_{s h}=a \cdot m_{v}+b,
$$

where $a$ and $b$ are constants at the given soil texture, frequency and incidence angle, $\Gamma_{s h}$ is the smooth land surface horizontal polarization reflectivity or Fresnel reflectivity. The two constants equal to 1.10 and 0.09 for a silt-loam soil with $30.6 \%$ sand, $55.9 \%$ silt and $13.5 \%$ clay at $1.4 \mathrm{GHz}$ and $30^{\circ}$ incidence angle [Yang et al., 2000]. These two constants will be updated from the comparison between TRMM/TMI estimated surface horizontal polarization Fresnel reflectivity and GAME/Tibet field-measured volumetric soil moisture in this study by assuming that the soil texture is homogeneous over the study area.

[12] The radiation scattering effects along the radiative transfer path cannot be eliminated above $5-10 \mathrm{GHz}$ in scattering media [Pampaloni and Paloscia, 1986]. When the scattering transfer is limited to forward direction, which can be described by Delta-Eddington approximation, the radiative transfer will take the simplified formulation as equation (1), only the single scattering albedo and optical thickness need to be revised as

$$
\begin{gathered}
\omega^{*}=(1-\alpha) \omega /(1-\alpha \omega) \\
\tau^{*}=(1-\alpha \omega) \tau,
\end{gathered}
$$

where $\alpha$ represents the fraction of radiation intensity scattered along incidence direction, $\alpha=0.05$ is assumed in this paper, $\omega$ and $\tau$ can be referred to literatures. As pointed out and validated by Van de Griend et al. [1996], the vegetation single scattering albedo and transmittance are different at horizontal and vertical polarization case, the difference should basically be due to plant water content, canopy structure and elements preferential orientation. However, the dual-polarized ratio of two parameters has almost no variation in the fixed incidence angle. The optimum values of these temporal invariant parameters in this study are from Van de Griend and Owe [1994], i.e., $\omega_{\mathrm{h}}=0.076, \omega_{\mathrm{v}}=0.063$ and $\tau_{\mathrm{h}}-\tau_{\mathrm{v}}=0.5108$. To better 
understand the computing processes of the proposed algorithm, a flowchart is given in Figure 1.

\section{Error and Sensitivity Analysis}

[13] The uncertainties of enrolled independent parameters can cause errors in determination of land surface soil moisture from TRMM/TMI data sets. To analyze magnitude of the error caused by these uncertainties, the partial derivative operator will be directly performed to equation (1) for the different parameters respectively. As soil moisture has a linear relationship with the estimated Fresnel reflectivity, Fresnel reflectivity hereby will be used as a soil moisture variable. The partial derivatives of soil reflectivity can be derived as following equations

$$
\begin{gathered}
\frac{\partial \Gamma_{p}}{\partial T_{B p}}=\frac{1}{T_{S} L\left(\omega^{2}-\omega-L\right)} \\
\frac{\partial \Gamma_{p}}{\partial L}=\frac{\omega \Gamma_{p}+2 L \Gamma_{p}-\omega-3 \omega L \Gamma_{p}}{\omega L^{2}-\omega L-L^{2}} \\
\frac{\partial \Gamma_{p}}{\partial \omega}=\frac{L+L^{2} \Gamma_{p}-L \Gamma_{p}-1}{\omega L+L^{2}-\omega L^{2}} \\
\frac{\partial \Gamma_{h}}{\partial \sigma}=2 \alpha \sigma(\beta+\varsigma) \Gamma_{s h v} \exp \left(-\beta \sigma^{2}-\zeta \sigma^{2}\right)-2 \zeta \sigma \Gamma_{s h} \exp \left(-\zeta \sigma^{2}\right) \\
-2 \zeta \alpha \sigma \Gamma_{s h v} \exp \left(-\zeta \sigma^{2}\right),
\end{gathered}
$$

where $\alpha=0.35, \beta=0.6 f, \zeta=\left(\frac{4 \pi v}{c} \cos ^{2} \theta\right), \Gamma_{\text {shv }}$ is the difference of horizontally and vertically polarized Fresnel reflectivity.

[14] Generally, if uncertainties of input parameters are introduced to the algorithm, the retrieved soil reflectivity will differ from the one retrieved with the true input parameters, here this difference (i.e., the difference between the retrieved soil reflectivity by using the perturbed parameters and that by using the reference ones) is defined as an error to assess the reliability of the proposed algorithm. To estimate the error magnitude, the typical values of every parameters in equations (11)-(14) are given as $\mathrm{T}_{\mathrm{s}}=280 \mathrm{~K}$, $\mathrm{L}=0.85, \Gamma_{\mathrm{h}}=0.15, \Gamma_{\mathrm{v}}=0.05$ and $\omega=0.1$ respectively. The calculation results show that one Kelvin uncertainty in brightness temperature will cause 0.0042 error to the soil reflectivity. The uncertainty of vegetation layer is characterized by the transmittance of the vegetation layer, $10 \%$ uncertainty in vegetation transmittance can give an error of 0.0125 for soil reflectivity. The single scattering albedo is an important parameter in radiative transfer process, it directly affects the partition of radiation scattering and absorption, when the value ranges from 0.04 to 0.20 for the general vegetation layer, $10 \%$ uncertainty in single scattering albedo will lead to 0.0230 unit error in estimated reflectivity. The reflectivity is very sensitive to surface roughness, especially at lower standard derivation of surface height. For the standard deviation of surface height around $0.35 \mathrm{~cm}$, error magnitude is about -0.01271 per $0.1 \mathrm{~cm}$ uncertainty.

[15] In order to have a holistic view of the reflectivity error range caused by the indeterminacy of above parameters, we normalize these enrolled parameters with their minimum and maximum values respectively the minimum and maximum values of surface temperature, vegetation attenuation factor, single scattering albedo and standard deviation of surface

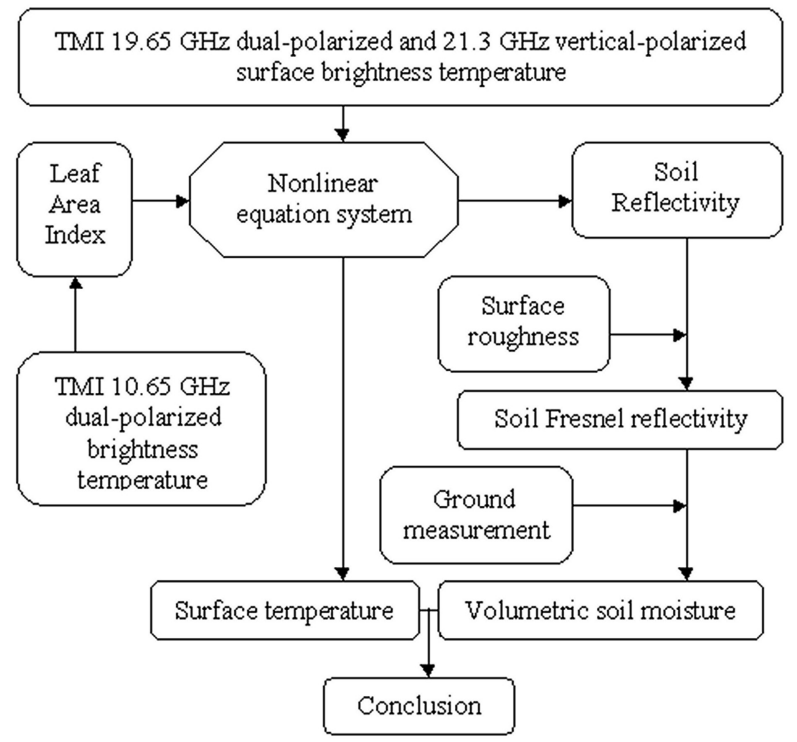

Figure 1. The flowchart for the determination of land surface temperature and soil moisture process from the TRMM/TMI data.

height are $265.0-325.0 \mathrm{~K}, 0.0-1.0,0.0-0.2$ and $0.25-0.65$ $\mathrm{cm}$. The results are plotted in Figure 2.

[16] It is shown in Figure 2 that the fluctuation of error are relatively steady at different brightness temperature and vegetation single scattering albedo, which is about 0.004 and 0.023 per Kelvin and $10 \%$ indeterminacy respectively. The soil reflectivity is very sensitive to vegetation attenuation factor when it is smaller than 0.5 which corresponds to a dense vegetation layer, the error gradually reduces to -0.0200 when transmittance is bigger than 0.5 over the sparsely vegetated land surface. The roughness has an obvious effect on soil reflectivity when land surface is relatively smooth. When the standard deviation of surface height is bigger than $0.35 \mathrm{~cm}$, the error in Fresnel reflectivity gradually decreases and will be close to the error magnitude caused by the uncertainty of brightness temperature. Fortunately, the normal ranges of the vegetation attenuation factor and standard deviation of the Tibetan plateau surface height are bigger than 0.5 and $0.35 \mathrm{~cm}$ in this study (i.e., the surface type can be characterized as sparsely vegetated rough surface).

[17] In summary, the uncertainty in the surface brightness temperature and single scattering albedo give very low error to the derived reflectivity, the vegetation attenuation factor also gives smaller error when it is greater than 0.5 (sparse vegetation). The reflectivity error is small when standard deviation of surface height is greater than $0.35 \mathrm{~cm}$, and will increase very quickly when standard deviation of surface height is lower than $0.35 \mathrm{~cm}$. As the magnitudes of the enrolled parameters fall within this range in most cases, the proposed algorithm will therefore be expected to give a reliable result over the Tibetan plateau.

\section{Study Area and Data Description}

[18] The study area is located in the central Tibetan Plateau among $75-95^{\circ} \mathrm{E} / 25-40^{\circ} \mathrm{N}$ in Eurasia continent. 


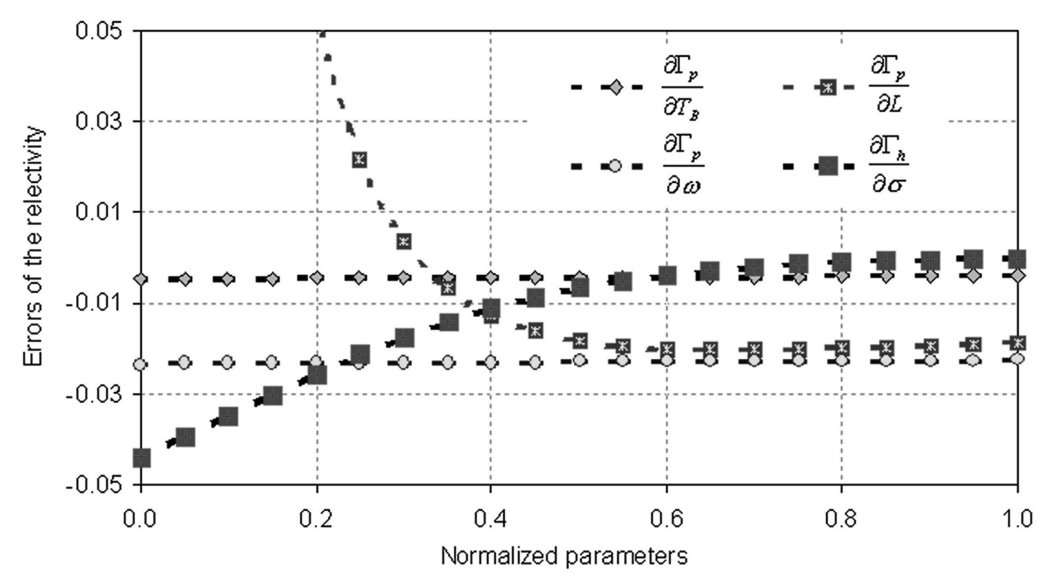

Figure 2. The error ranges of land surface soil reflectivity caused by uncertainties of the enrolled parameters. The uncertainties of brightness temperature is one Kelvin, 0.1 unit vegetation attenuation factor and single scattering albedo, $0.1 \mathrm{~cm}$ standard deviation of surface height.

Because of the special geographic landscape, high elevation and strong local convective cloudy weather characteristics, it is almost impossible to continuously evaluate the regional and temporal distribution of land surface variables with optical and infrared remote sensing data over the Tibetan plateau. Although ground measurements can be conducted at point, manpower cannot reach most of the Tibetan plateau area because of the severe natural circumstance, it is also impossible to directly obtain the reliable regional distribution of soil moisture and vegetation with such point measurements. Therefore the land surface variable can only be measured satisfactorily from space techniques. In this contribution, it will be demonstrated that microwave remote sensing can bridge insufficiency of the optic and infrared remote sensing in the determination of land surface variables. Data collected during the Global Energy and Water Experiment (GEWEX) Asian Monsoon Experiment in Tibet (GAME/Tibet), in particular the soil volumetric soil moisture and land surface temperature measured during Intensive Observation Period in 1998 (IOP'98) field campaign are useful in this study.

[19] Nine observation sites (Tutuohe, D110, WADD, NODA, Anduo, MS3478, Naqu, MS3608 and MS3637) are available for the land surface temperature and seven for soil moisture measurements (Soil moisture was not recorded at Naqu site and was missing at WADD) recorded by the Soil Moisture and Temperature Measurement System (SMTMS) installed along the north-south transect, their

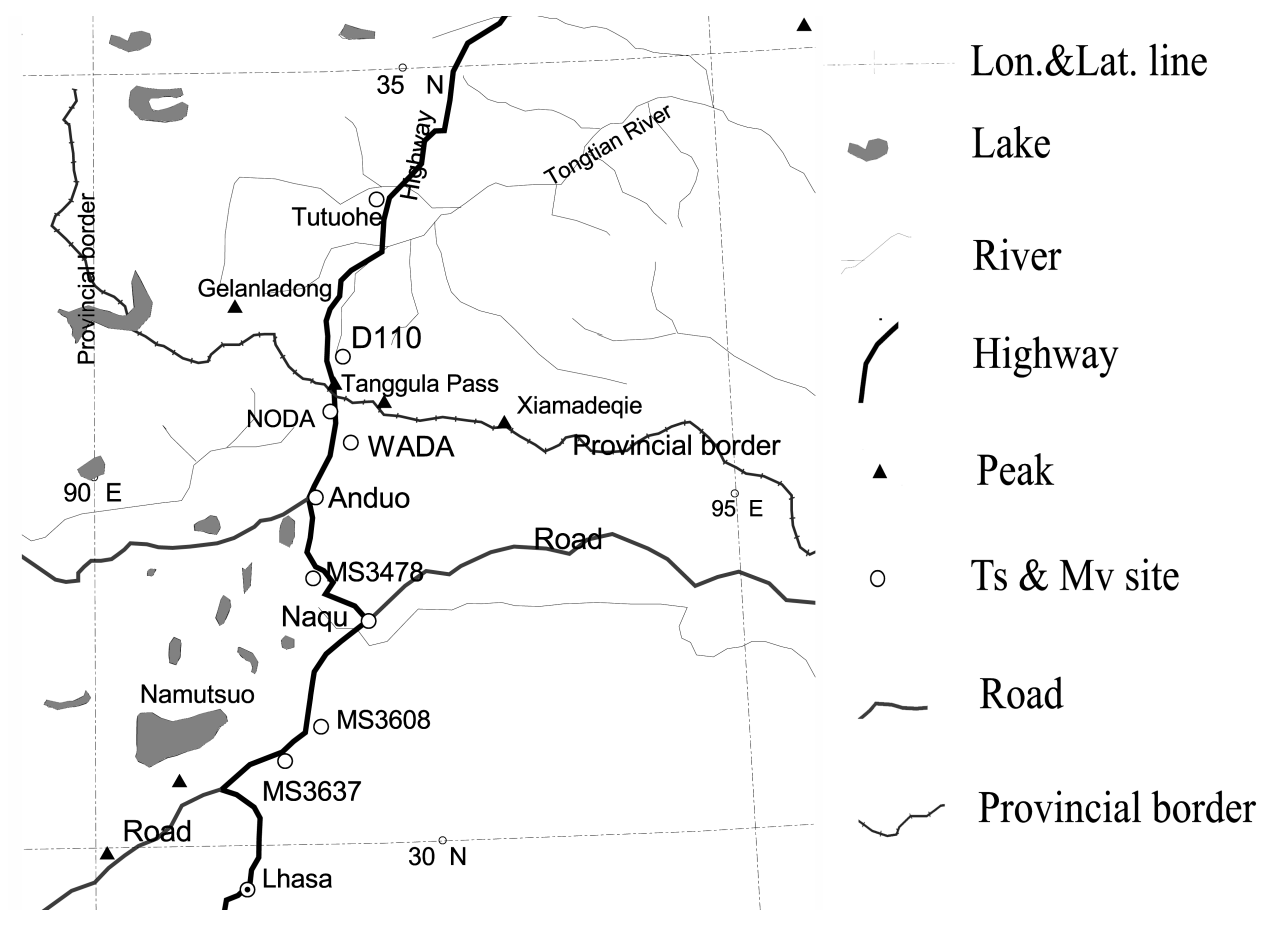

Figure 3. The detailed location and distribution of the GAME/Tibet field campaign sites covered by the available TRMM/TMI data in this study. 


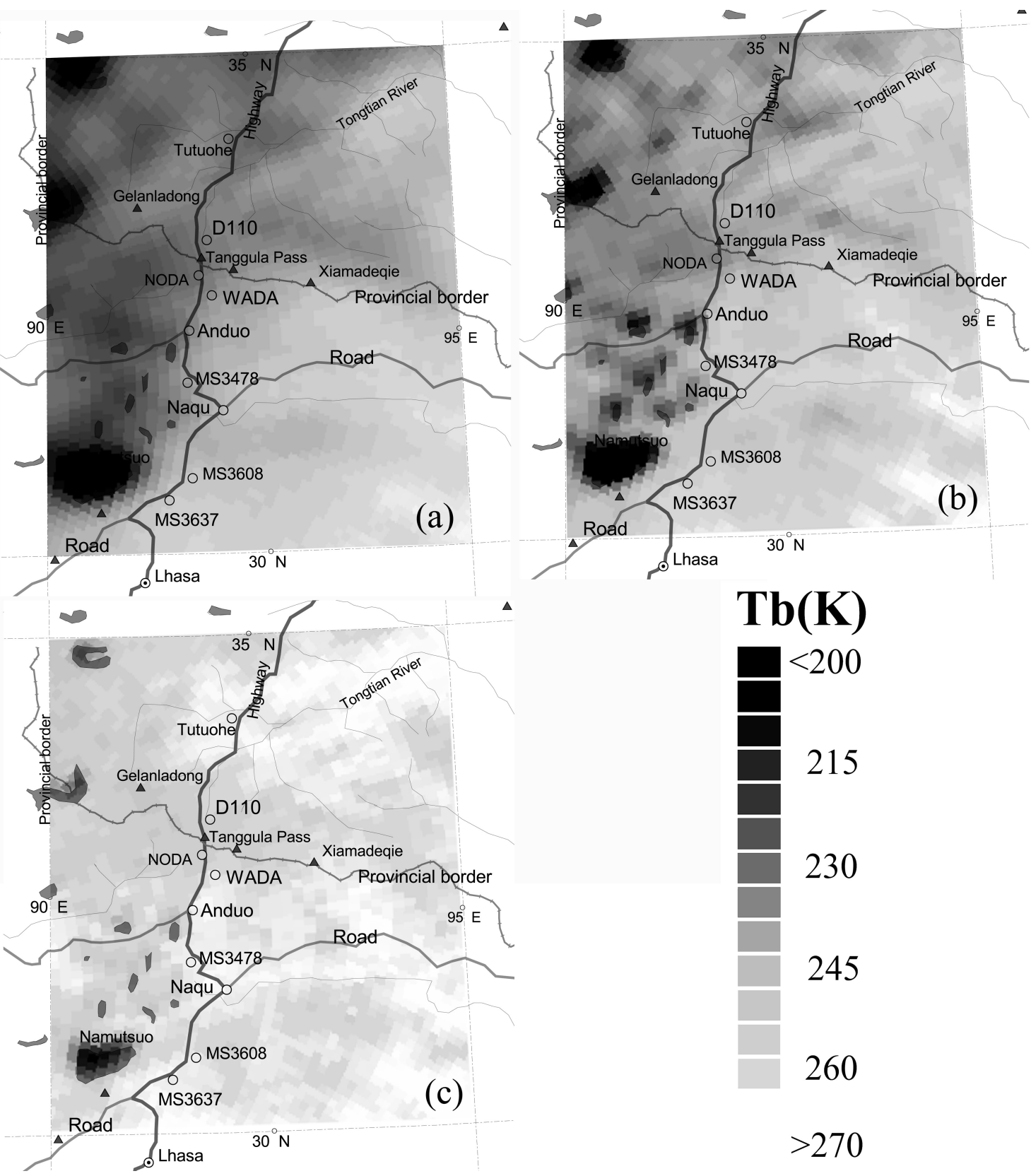

Figure 4. The regional distribution of TRMM/TMI brightness temperature over the study area, 14:54 GMT, 13 September 1998: (a) $10.5 \mathrm{GHz}$ horizontal polarization, (b) $19.0 \mathrm{GHz}$ horizontal polarization, (c) $21.4 \mathrm{GHz}$ vertical polarization.

location and distribution are plotted in Figure 3. These sites were selected by the GAME/Tibet SMTMS team to represent the average situation on the Tibetan central area. Other relevant data are recorded by the planetary boundary layer towers and automatic weather stations at these sites.

[20] The ground surfaces of all sites were covered with sparse short-grass with leaf area index smaller than 0.5 . The canopy heights were about $0.03 \mathrm{~m}$ in the first ten days of September 1998. The fraction of the ground covered by vegetation was approximately 0.3 during this period. The soil type was predominately a sandy silt loam with small pebbles. At each site soil temperature was measured at the depths of $0.04,0.2,0.4,0.6,0.8,1.0,1.3,1.6$ and $2.0 \mathrm{~m}$ below the surface by the temperature sensor and the DATAMARK-LS3000 data logger, while soil moisture was measured at depths of $0.04,0.2,0.6,1.0,1.6$ and $2.0 \mathrm{~m}$ with the TRIME-MUX6 and the same type of data logger as for the temperature measurements. The recorded data show that soil temperature below $0.6 \mathrm{~m}$ depth is almost constant. The volumetric soil moisture was measured every ten minutes, half-hour or hour at the different sites. The measured soil moisture is assumed to represent the local ground truth in this study. Surface temperature used for the comparison was recorded with the infrared radiometers together with other micrometeorological measurements and was recorded automatically as half hourly averages. Because of the extensive 
spatial-temporal coverage of field sites, the collected ground measurement data are especially suited for the validation of satellite remote sensing retrieval algorithms.

[21] The detailed locations of the GAME/Tibet campaign field sites covered by the available TRMM/TMI data are shown in Figure 3. Land surface types of the study area consist of mountains, river basins, water bodies, grassland and bare soil surfaces. There are several lakes, among which a large one is Namutso, in the southwest of the selected area. Tanggula Mountain crosses the middle of selected area, which is also the administrative border between Qinghai province and Tibet autonomous region; Nianqin Tanggula Mountain crosses $31-32^{\circ} \mathrm{N}$ regions. Some areas with permanent frozen soil or snow covers exist around mountainous area. The elevation of the southeastern area is relative high with high vegetation fractional cover. Tongtianhe and Tuotuohe river basins, many small branches of these rivers and marshland are distributed in the northeastern area.

[22] The Tropical Rainfall Measuring Mission (TRMM) is the first space mission to quantitatively measure tropical rainfall from space. TRMM Microwave Imager (TMI) provides continuous observations of ocean and land surface in several microwave bands with $5-63 \mathrm{~km}$ spatial resolution. The collected TRMM/TMI data covered an area from $90-95^{\circ} \mathrm{E}$ and $30-35^{\circ} \mathrm{N}$; twelve TRMM/TMI overpasses in September 1998 are available to this study over the GAME/ Tibet IOP'98 mesoscale experimental area. The overpass dates are: 1, 2, 4, 6, 8, 11, 13, 15, 17, 22, 25, and 27 September 1998.

[23] Rainfall has effects on TRMM/TMI observed brightness temperature and the retrieved soil moisture as well during rainfall events. However, the rainfall events become less frequent from September on the Tibetan plateau because it is after the Monsoon season. Since the most TRMM/TMI overpasses data did not overlay with the rainfall events in this study, the rainfall effects on brightness temperature observation will not be considered in this study. Land surface temperature and volumetric soil moisture measured during GAME/Tibet can be used to validate the proposed method by comparing with TRMM/TMI retrieved surfaces temperature and soil moisture. The regional distributions of original brightness temperature over the selected area are shown in Figure 4. It reveals that the brightness temperature of different channel exhibits a regional distribution; the large water bodies can be readily identified because of the lower brightness temperature. However, brightness temperature at a single channel is insufficient as direct information to infer land surface temperature and soil moisture because it is an integrated response of the land surface temperature, soil moisture and roughness. Thus with the algorithm based on the radiative transfer process and an advanced numerical solution of nonlinear equations system, it is expected to estimate land surface temperature and soil moisture over the central Tibetan plateau.

\section{Land Surface Temperature and Soil Moisture Over the Tibetan Plateau}

[24] The land surface temperature and soil Fresnel reflectivity are evaluated both at regional and pixel scale over the
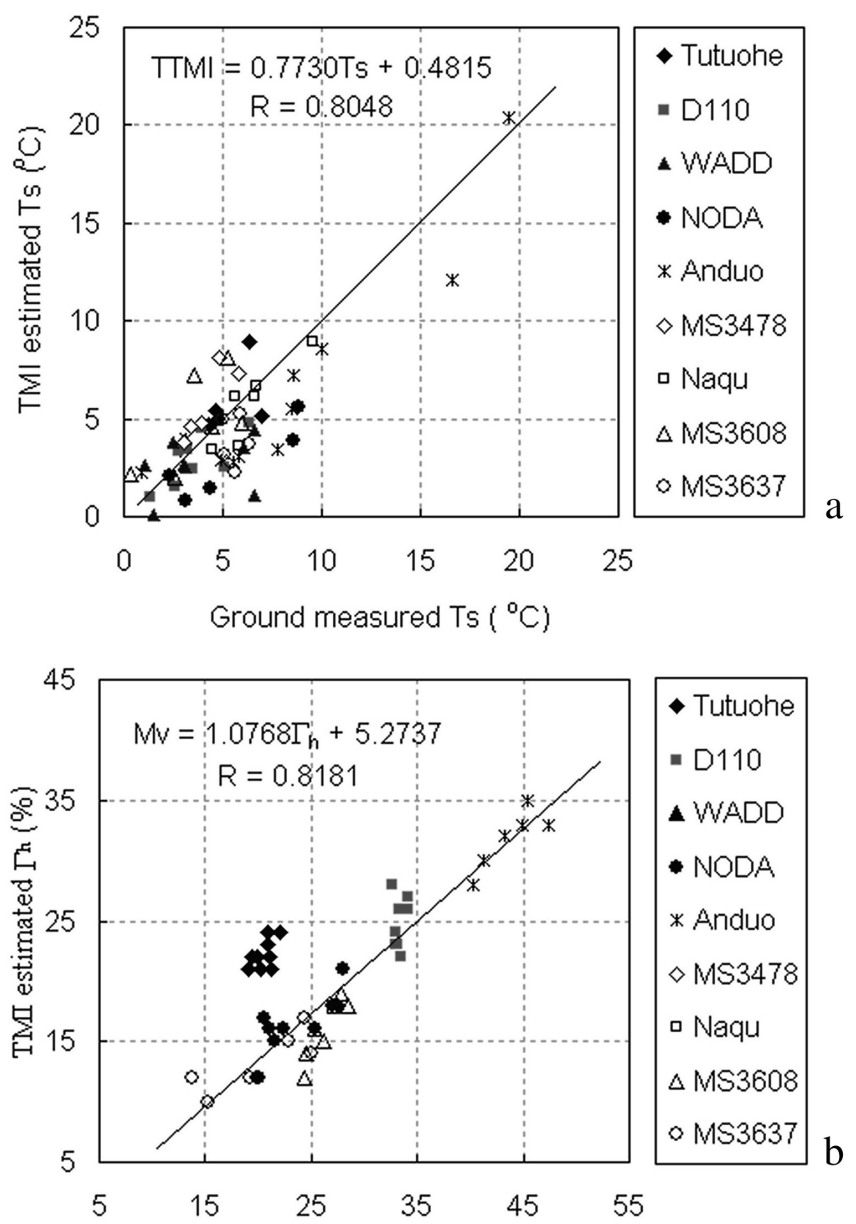

Figure 5. The comparison between the ground measurements and TRMM/TMI estimates: (a) surface temperature, (b) volumetric soil moisture.

selected area with the proposed algorithm and collected TRMM/TMI data in this section. To validate the proposed algorithm, the ground-measured land surface temperature and volumetric soil moisture most close to TRMM/TMI overpass times (different for each overpass) are extracted from GAME/Tibet database. The comparison results are presented in Figure 5. Since the available TRMM/TMI scenes did not cover all the validation sites in the overpasses, and some ground-measured soil moisture were not available or missing for some overpasses at some sites, the total sample number available for comparison of soil moisture is not as much as for temperature in Figure 5. In particular, the ground measurements of soil moisture were not available after 17 September 1998, and there was also no ground soil moisture observation at Naqu but only land surface temperature.

[25] It can be seen that the ground-measured surface temperature is in good agreement with TRMM/TMI estimated land surface temperature (Figure 5a). The average difference is $0.52 \pm 2.41 \mathrm{~K}$ for 55 samples. A quasi-linear relationship exists between ground-measured volumetric soil moisture and TRMM/TMI estimated soil Fresnel reflectivity, the correlation coefficient reaches 0.80 for these available samples. Figure 5b also shows that TRMM/TMI estimated Fresnel reflectivity values are systematically 

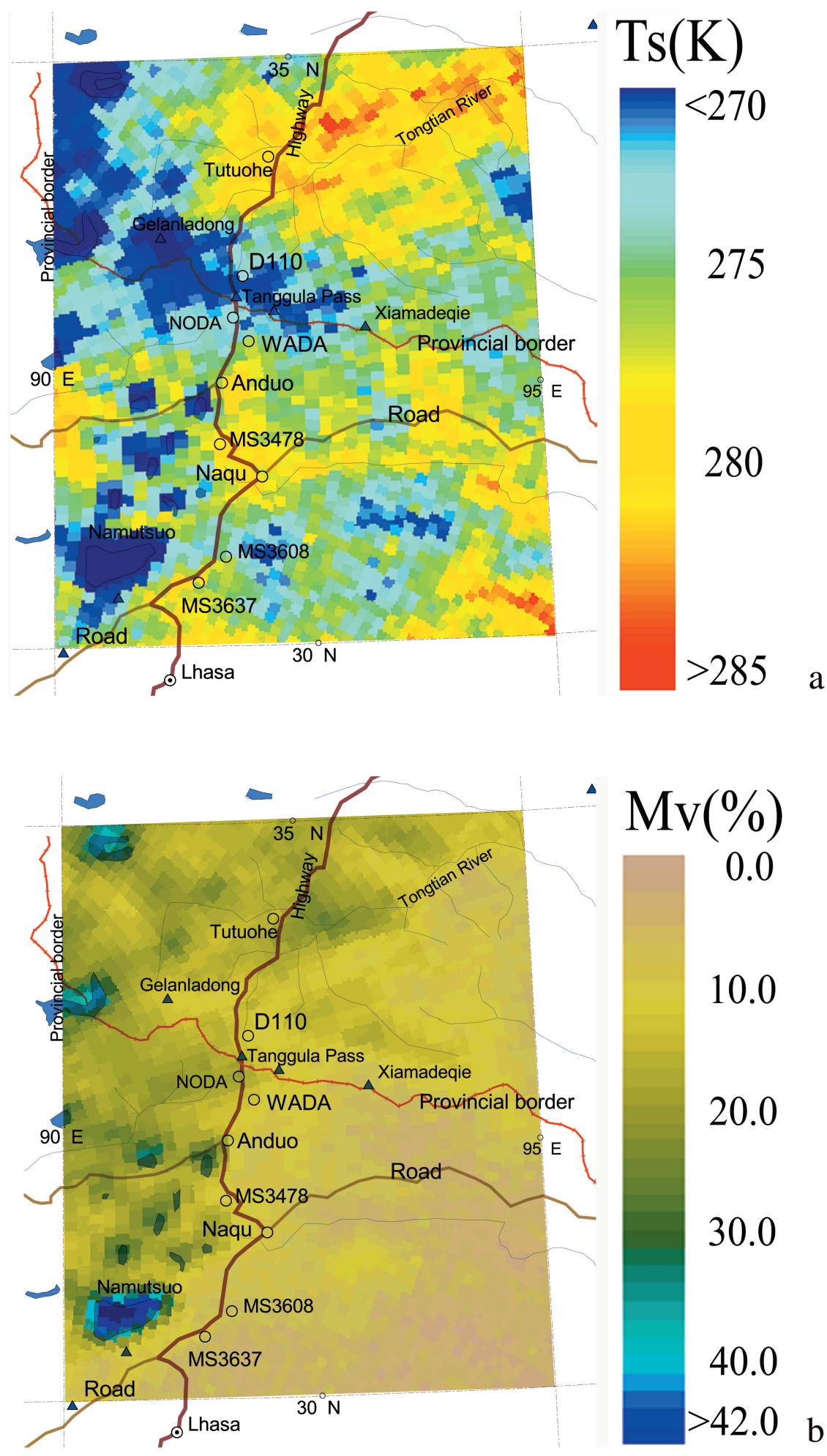

Figure 6. The regional distribution of the estimated land surface temperature and volumetric soil moisture estimated over the GAME/Tibet experimental area, 14:54 GMT, 13 September 1998.

higher at Tuotuohe site when compared to the other observation data. The reason is that Tutuohe ground observation site is close to Tuotuo river, TRMM/TMI observation has integrated water body's information into the observation data due to its coarse resolution while the ground measure- ment only takes into account the local soil information, this will lead a systematic shift between the ground measurement and satellite retrieved result. If these observations at Tuotuohe sites are rejected, the relationship between the ground-measured temperature and TRMM/TMI estimated 
surface temperature will be much improved. As the spatial resolution of TRMM/TMI is rather coarse, the comparison between satellite remote sensing retrieved results and the ground measurements are still questionable in most cases. Nevertheless, since the geographic locations of field campaign sites were carefully chosen to ensure that these field sites are regionally representative, TRMM/TMI retrieved results can be judged very successful.

[26] The regional distribution images of land surface temperature and soil moisture are mapped with the data collected when TRMM/TMI overpass of the study area at 14:54 GMT, 13 September 1998 (Figure 6). The estimated surface temperature ranges $265-285 \mathrm{~K}$, volumetric soil moisture ranges $4.0-50.0 \%$. (Figure 7, cf. Figure 3). Tanggula Mountainous area, which is the provincial border of Qinghai province and Tibet autonomous region, Nianqing Tanggula area, which are snow covered or with permanent frozen soil and several lakes, exhibit low surface temperature below $273.0 \mathrm{~K}$. Although the algorithm is questionable over snow cover, water and frozen soil surface because of their different dielectric behaviors when compared to moist soils, it still successfully retrieved lower surface temperatures over these areas. The area around Gladandong peak and the peak beside Namutsuo Lake can be identified because of the lower surface temperatures. Tongtian and Tuotuo river basins exhibit higher land surface temperature compared to mountainous areas. As TRMM/TMI overpasses the study area in late evening in this scene, the retrieved land surface temperature is generally relative low.

[27] TRMM/TMI estimated soil moisture field reveals that the southeastern part of study area gives lower soil moisture values, while the lake areas and river basin have higher soil moisture. Together with the comparison of surface temperature and soil moisture between TRMM/ TMI estimates and ground measurements as presented in Figure 5, these results, taking into consideration of TRMM/ TMI spatial resolution and regional representative characteristics of ground observation sites, reflect the real thermal and wetness status over the study area.

[28] The histogram of the estimated land surface temperature ranges in $265-285 \mathrm{~K}$ with a peak around $276 \mathrm{~K}$ for the overpass at 14:54 GMT, 13 September 1998 (Figure 7). The estimated volumetric soil moisture ranges $4.0-50.0 \%$ with two peaks around $8.0 \%$ and $20.0 \%$, which should correspond to the dominant area for dry soil and wetter areas in the study area. If the volumetric soil moisture is higher than $40 \%$, the corresponding land surface should be considered as saturated surface soil or water bodies in this case.

[29] The regional distribution patterns of the TRMM/TMI estimated land surface temperature and soil moisture from different overpasses reveal similar characteristics although the atmospheric status is different at each overpass. This confirms that atmosphere and cloud have less attenuation effects on microwave radiative transfer process if no rainfall events were occurring simultaneously. All these results demonstrate that, the proposed algorithm is successful to evaluate surface temperature and soil moisture simultaneously from TRMM/TMI remote sensing data. Nevertheless, as a complementary remark, the proposed method still needs to be improved in several aspects. The surface roughness parameterization is still a problem, which is not directly evaluated from TRMM/TMI data sets, but from a previous

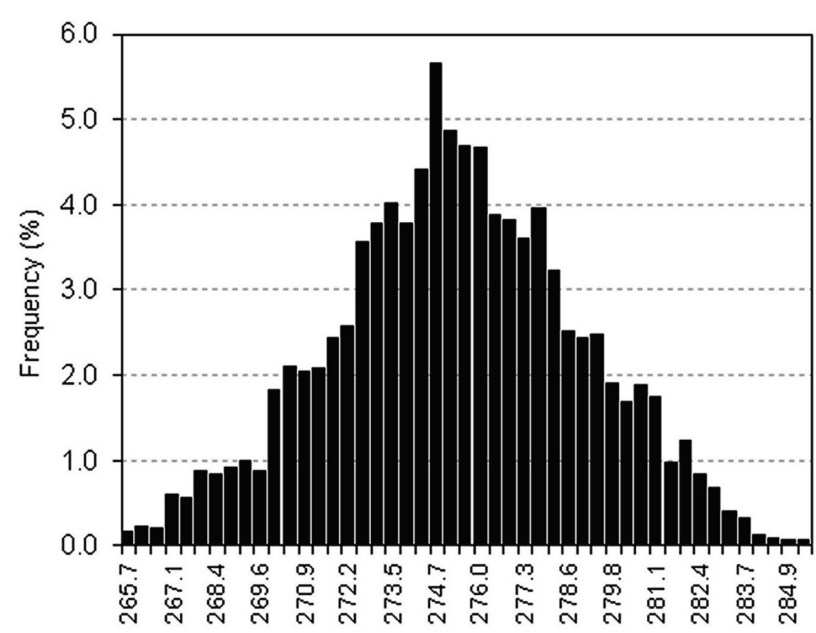

Land surface temprature with 0.4247 bin size $(\mathrm{K})$

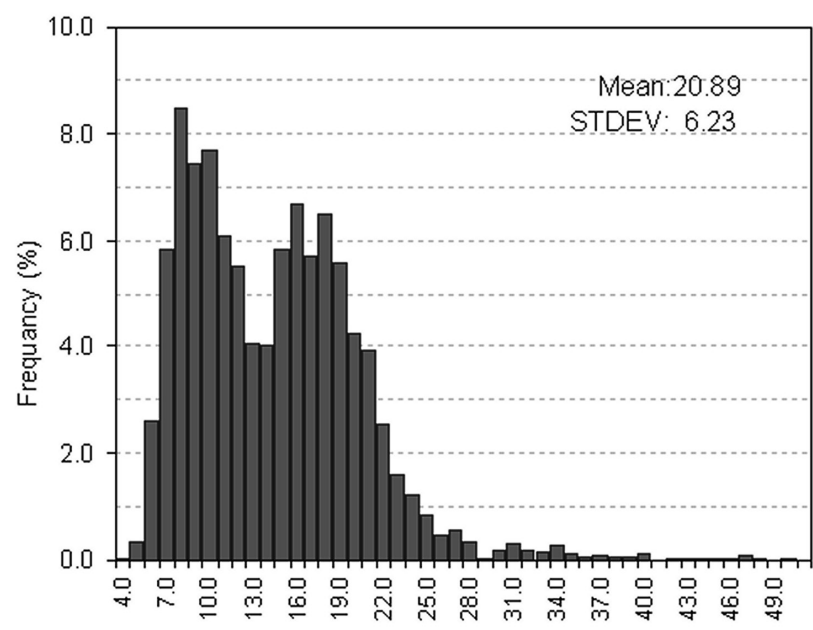

Volume soil moisture with one percent bin size (\%)

Figure 7. The histogram of the estimated land surface temperature and volumetric soil moisture over the GAME/ Tibet experimental area, 14:54 GMT, 13 September 1998.

study. The effects of the vegetation layer also need to be carefully considered. Furthermore, it is desirable that the proposed method could be validated with ground data from other field experiments.

\section{Conclusion}

[30] Based on the microwave radiative transfer equations and the TRMM/TMI observation data at lower frequency channels, an algorithm for the determination of land surface temperature and soil moisture has been developed in this paper. With the proposed algorithm and the TRMM/TMI lower frequency data, the land surface temperature and soil moisture has been retrieved both at point and regional scale. To validate the proposed algorithm, the ground-measured surface temperature and volumetric soil moisture data were used which were collected during the GAME/Tibet field experiment in 1998. 
[31] The TRMM/TMI estimated land surface temperature is in good agreement with the ground-measured surface temperature, the average difference is $0.52 \pm 2.41 \mathrm{~K}$ with a correlation coefficient of 0.80 . A linear relationship exists between ground-measured volumetric soil moisture and the estimated soil Fresnel reflectivity with a correlation coefficient of 0.82 . The estimated surface temperature and soil moisture is in good agreement with GAME/Tibet field observation data not only in the regional and temporal distribution but also in their statistical characteristics. The regional distribution shows that the estimated land surface temperature gives lower values in mountainous areas, while the river basins have higher surface temperature. The retrieved volumetric soil moisture exhibits lower values in southeast area, while the areas adjacent to lakes located in the west of the study area and river basins have high soil moisture values. Therefore the proposed algorithm is successful in the retrieval of the land surface thermal and wetness status over the study area.

[32] Acknowledgments. This work was performed under sponsorship by the Netherlands Remote Sensing Board (BCRS), the GIS/RS program of the Dutch Ministry of Agriculture, Nature and Fishery (LNV), the Netherlands Royal Academy of Arts and Sciences, and the auspices of the Chinese National Key Programmme for Developing in Basic Sciences (G1998040900) and the Innovation Project of Chinese Academy of Sciences (KZCX2-301). Many thanks are due to the TRMM/TMI data provider. The authors are grateful to two anonymous reviewers for their constructive comments.

\section{References}

Asrar, G., R. B. Myneni, Y. Li, and E. T. Kanemasu, Measuring and modeling spectral characteristic of tall grass prairie, Remote Sens. Environ., 27, 143-155, 1989.

Becker, F., and B. J. Choudhury, Relative sensitivity and microwave polarization difference index for vegetation and desertification monitoring, Remote Sens. Environ., 24, 297-331, 1988.

Calvet, J.-C., A. Chanzy, and J.-P. Wigneron, Surface temperature and soil moisture retrieval in the Sahel from multiple frequency radiometry, IEEE Trans. Geosci. Remote Sens., 34, 588-600, 1996.

Chanzy, A., and J.-P. Wigneron, Microwave emission from soil and vegetation, COST Action 712, EUR 19 543, 89-103, Radiative Transfer Models for Microwave Radiometry Eur. Coop. in the Field of Sci. and Tech. Res. Meteorol., Eur. Comm., Geneva, 2000.

Choudhury, B. J., T. J. Schmugge, A. Chang, and R. W. Newton, Effect of surface roughness on microwave emission from Soil, J. Geophys. Res., 84, 5699-5706, 1979.

Fujii, H., and T. Koike, Development of a TRMM/TMI algorithm for precipitation in the Tibetan Plateau by considering effects of land surface emissivity, J. Meteorol. Soc. Jpn., 79, 475-483, 2001.

Kerr, Y. H., and E. G. Njoku, A semi-empirical model for interpreting microwave emission from semi-arid land surface as seen from space, IEEE Trans. Geosci. Remote Sens., 28, 384-393, 1990.

Matzler, C., and A. Wismann, Surface emissivity data from microwave radiometer experiments of the University of Bern, Appendix A, Radiative transfer models for microwave radiometry, COST Action 712, EUR 19543, pp. $163-172$, Eur. Coop. in the Field of Sci. and Tech. Res. Meteorol., Eur. Comm., Geneva, 2000.

Njoku, E. G., and J. A. Kong, Multifrequency microwave radiometer measurements of soil moisture, J. Geophys. Res., 82, 3108-3118, 1977.

Ormsby, J. P., and B. J. Choudhury, Vegetation spatial variability and its effect on vegetation indices, Int. J. Remote Sens., 8, 1301-1306, 1987.

Paloscia, S., G. Macelloni, E. Santi, and T. Koike, A multifrequency algorithm for the retrieval of soil moisture on large scale using microwave data from SMMR and SSM/I satellites, IEEE Trans. Geosci. Remote Sens., 39, 655-1661, 2001.

Pampaloni, P., and S. Paloscia, Microwave emission and plant water content: A comparison between field measurements and theory, IEEE Trans. Geosci. Remote Sens., 24, 900-905, 1986.

$\mathrm{Su}, \mathrm{Z}$., Remote sensing of land use and vegetation for mesoscale hydrological study, Int. J. Remote Sens., 21, 213-333, 2000.

Tadono, T., T. Koike, K. Arida, and J. Shi, Validation of SAR algorithm for soil moisture mapping in the Tibetan Plateau, in Proceedings of 1st International Workshop on GAME/Tibet, edited by A. Numaguti, L. Liu, and L. Tian, pp. 51-54, Chin. Acad. of Sci./Jpn. Natl. Comm. for GAME, Xi'an, China, 1999.

Tucker, C. J., and P. J. Sellers, Satellite remote sensing of primary production, Int. J. Remote Sens., 7, 1395-1416, 1986.

Ulaby, T., R. K. Moore, and A. K. Fung, Microwave Remote Sensing, vol. 2, Radar Remote Sensing and Emission Theory, Addison-Wesley-Longman, Reading, Mass., 1982.

Van de Griend, A. A., and M. Owe, The influence of the polarization on canopy transmission properties at $6.6 \mathrm{GHz}$ and implications for large scale soil moisture monitoring in semi-arid environments, IEEE Trans. Geosci. Remote Sens., 32, 409-415, 1994.

Van de Griend, A. A., M. Owe, J. de Ruiter, and B. T. Gouweleeuw, Measurement and behavior of dual-polarization vegetation optical depth and single scattering albedo at $1.4 \mathrm{GHz}$ microwave frequency, IEEE Trans. Geosci. Remote Sens., 34, 957-965, 1996.

Wang, J. R., and B. J. Choudhury, Remote sensing of soil moisture content over bare field at $1.4 \mathrm{GHz}$ frequency, J. Geophys. Res., 86, 5277-5282, 1981.

Wen, J., and Z. Su, Active microwave remote sensing on soil moistureAlgorithms for ERS Wind sactterometer data, in Active Microwave Remote Sensing of Soil Moisture for Initialization of Numerical Weather Prediction 2001, edited by Z. Su and G. J. Roerink, Rep. USP-2/01-04, pp. 27-40, User Support Prog., Neth. Remote Ses. Board, Delft, 2001.

Wigneron, J. P., Y. H. Kerr, A. Chanzy, and Y. Q. Jin, Inversion of surface parameters from passive microwave measurements over a soybean field, Remote Sens. Environ., 46, 61-72, 1993.

William, H. P., P. F. Brian, and A. T. Saul, Numerical Recipes in C: The Art of Scientific Computing, 2nd ed., Cambridge Univ. Press, New York, 1992.

Yanai, M., and C.-F. Li, Seasonal heating of the Tibetan Plateau and its effects on the evaluation of the Asian summer monsoon, J. Meteorol. Soc. Jpn., 70, 319-351, 1994

Yang, D., F. T. Ulaby, and M. C. Dobson, Sensitivity to soil moisture by active and passive microwave sensors, IEEE Trans. Geosci. Remote Sens., 38, 105-114, 2000.

Y. Ma, Cold and Arid Regions Environmental and Engineering Research Institute, Chinese Academy of Sciences, Lanzhou, China. (ymma@ns.lzb. ac.cn)

Z. Su and J. Wen, Wageningen University and Research Centre, Alterra Green World Research, P.O. Box 47, NL-6700 AA Wageningen, Netherlands. (B.Su@alterra.wag-ur.nl; J.Wen@Alterra.wag-ur.nl) 